Sammlung Metzler

Band 304 
Regina Fasold

\section{Theodor Storm}

Verlag J.B. Metzler

Stuttgart - Weimar 
Die Deutsche Bibliothek - CIP-Einheitsaufnahme

Fasold, Regina:

Theodor Storm / Regina Fasold.

Stuttgart ; Weimar : Metzler, 1997

(Sammlung Metzler ; Bd. 304)

ISBN 978-3-476-10304-8

ISBN 978-3-476-10304-8

ISBN 978-3-476-05158-5 (eBook)

DOI 10.1007/978-3-476-05158-5

ISSN $0558-3667$

\section{SM 304}

Dieses Werk einschließlich aller seiner Teile ist urheberrechtlich geschützt. Jede Verwertung außerhalb der engen Grenzen des

Urheberrechtsgesetzes ist ohne Zustimmung des Verlages unzulässig und strafbar. Das gilt insbesondere für

Vervielfältigungen, Übersetzungen, Mikroverfilmungen und die Einspeicherung und Verarbeitung in elektronischen Systemen.

(C) 1997 Springer-Verlag GmbH Deutschland

Ursprünglich erschienen bei J.B. Metzlersche Verlagsbuchhandlung und Carl Ernst Poeschel Verlag GmbH in Stuttgart 1997 


\section{Inhalt}

Vorwort

I. Biographie

1. Kindheit und Schulzeit (1817-1837) ................................. 1

2. Studium in Kiel und Berlin (1837-1842) ......................... 10

2.1 Bertha von Buchan .................................................. 13

3. Husum (1843-1853) ............................................... 16

3.1 Die schleswig-holsteinische Erhebung;

Storm und die Politik ................................................ 23

4. Potsdam (1853-1856) ................................................ 28

5. Heiligenstadt $(1856-1864)$............................................. 35

6. Husum (1864-1880) ........................................................ 46

6.1 Politische Entwicklungen ........................................... 46

6.2 Familiäre Krisen ................................................... 49

6.3 Weltanschauliche Entwicklung, künstlerische

Krise und Neuansatz .............................................. 57

7. Hademarschen $(1880-1888)$............................................... 61

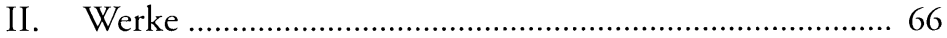

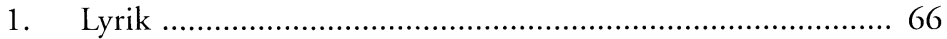

1.1 Überblick über das lyrische Schaffen;

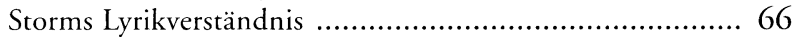

1.2 Persönlichkeitsstruktur und lyrische Stimmungen ‘............. 69

1.3 Forschungslage zur Lyrik ............................................ 79

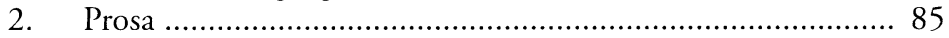

2.1 Frühe Prosa (1848-1853) ......................................... 85

2.1.1 Traditionen und Poetik .................................... 85

2.1.2 Erinnerungsperspektive, der Forschungsstand ......... 93

2.1.3 Immensee $(1850)$............................................. 95

2.2 Novellistik in Potsdam (1853-1856) ............................. 98

2.3 Heiligenstädter Novellistik (1856-1864) ....................... 101

2.3.1 Auf dem Staatshof (1859) ................................. 102

2.3.2 Im Schloß (1862), Veronica (1861) ....................... 105

2.3.3 Auf der Universität (1863) und andere Novellen .... 107 
2.4 Spukgeschichten und Märchen .............................. 111

2.5 Späte Novellistik (1867-1888) ................................ 116

2.5.1 Der Überblick über das Spätwerk in der

Forschungsliteratur (Einführungen und

Gesamtdarstellungen) ..................................... 116

2.5.2 Storms konzeptionelle Überlegungen zu seiner späten Novellistik ........................................... 121

2.5.3 Von In St. Jürgen (1868) bis Im Nachbarhause links (1875) ................................................. 127

2.5.4 Aquis submersus (1876) ................................... 134

Exkurs: Chroniknovellen ............................................ 140

2.5.5 Carsten Curator (1878), Der Herr Etatsrat (1881), Hans und Heinz Kirch (1882), Ein Doppelgänger (1886) - Der Eltern-Kind-Konflikt in Storms späten Novellen ............................................. 143

2.5.6 Der Schimmelreiter (1888) .............................. 152

III. Materialien .............................................................. 168

1. Der handschriftliche Nachlaß ....................................... 168

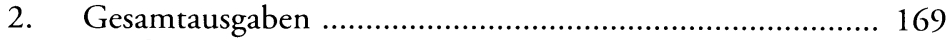

3. Briefe ................................................................. 171

4. Bibliographische Hilfsmittel, Forschungsberichte ............. 173

4.1 Bibliographische Hilfsmittel .................................. 173

4.2 Forschungsberichte .............................................. 174

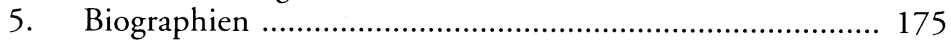

Abkürzungsverzeichnis .................................................. 178

Bibliographie ................................................................... 181

1. Materialien ............................................................. 181

2. Sekundärliteratur ..................................................... 186

Personenegister ................................................. 218

Angaben zur Autorin ........................................... 222 


\section{Vorwort}

Der vorliegende Band baut auf frühere Forschungsberichte auf und berücksichtigt vorrangig die letzten 25 Jahre der wissenschaftlichen Beschäftigung mit Theodor Storm, die hier erstmals resümiert werden. Bestimmte Schwerpunktsetzungen innerhalb der Storm-Forschung der zurückliegenden Jahrzehnte sind jedoch nur verständlich, wenn die Rezeptionsgeschichte des Autors vor und unmittelbar nach 1945 nicht aus dem Blick gerät. Auf der Grundlage der bereits im 19. Jahrhundert beginnenden Herauslösung der Stormtexte aus dem historischen, geistesgeschichtlichen und poetologischen Bezugshorizont, so konstatierte es Vinçon 1973, war letztlich eine partielle faschistische Funktionalisierung des Autors möglich, die u.a. der wichtige Storm-Biograph Franz Stuckert mit zu verantworten hatte. Gerade seine Arbeiten, in den interpretatorischen Teilen nur leicht durch eine existentialistisch anmutende Sicht überformt, prägten noch nach 1945 das Stormbild in ganz entscheidender Weise. Vinçon hatte 1973 aus dieser spezifischen Rezeptionsgeschichte heraus, deren differenzierte Darstellung freilich noch aussteht, seinen rigoros vertretenen ideologiekritischen Ansatz begründet. Auch sonst war die deutsche Wissenschaft - voran die Storm-Gesellschaft und ihr langjähriger Sekretär und nunmehriger Präsident Karl Ernst Laage seit Ausgang der 60er Jahre dezidiert darum bemüht, Autor und Werk aus dem Kontext von Literatur-, Sozial- und Politgeschichte zu erklären sowie im Zusammenhang mit Entwicklungen in Philosophie, Religionskritik und Naturwissenschaft in der 2. Hälfte des 19. Jahrhunderts zu sehen. Hauptaugenmerk galt dabei den gesellschaftskritischen Potentialen von Lyrik und Novellistik. Die Verdienste Peter Goldammers, der seit den 50er Jahren in der DDR die Stormforschung gleichsam repräsentierte und der die wichtige, über Jahrzehnte neben Kösters Ausgabe für wissenschaftliche Zwecke einzig geeignete Edition der Werke des Autors vorlegte, seien an dieser Stelle besonders hervorgehoben.

Seit den 80er Jahren kann man eine erfreuliche Ausdifferenzierung der Deutungsansätze auch an den Interpretationen der StormNovellen beobachten, so daß sich in der Darstellung eine Verknüpfung und synthetisierende Zusammenführung von z.T. recht heterogenen Auffassungen notwendig macht. 
Der Band gibt sowohl eine Einführung in die Biographie des Autors als auch einen Überblick über die Werkentwicklung mit den wichtigsten Forschungsresultaten. Jedoch erfolgt damit keine erlebnisästhetische Projektion von biographischen Daten auf die literarischen Texte. Vielmehr soll in den interpretatorischen Passagen des Bandes vor allem auf die Kontinuitäten innerhalb der Werkgenese aufmerksam gemacht werden. In der Novellistik betrifft das die Entfaltung und Variation von Erzähltechnik und Kernmotivik, in der Lyrik die zentralen 'Stimmungen`. Deshalb bilden die Kapitel zur Poetik von Lyrik und früher Novellistik, Kap. II.1.2. bzw. II.2.1., die in engem Zusammenhang mit Überlegungen zur Persölichkeitsstruktur des Autors entwickelt wurden, Ausgangs- und Bezugspunkt für die Deutungsansätze zu einzelnen Novellen, in deren Mittelpunkt der persönliche Mythos des Autors im Sinne Charles Maurons steht.

Bei der Erörterung des Spätwerks (1864-1888) sind nicht alle der 29, z.T. in ihrer ästhetischen Qualität recht umstrittenen Novellen berücksichtigt worden, sondern im wesentlichen nur jene, die auch in der wissenschaflichen Betrachtung einen Schwerpunkt bilden.

Das Stormbild wurde und wird in diesem Lande sehr wesentlich durch die Deutschlehrer mitgeprägt. Ein spezieller und sehr wichtiger Strang der Rezeptionsgeschichte erforderte deshalb eine gesonderte Untersuchung, die hier nicht geleistet werden konnte: die für Lehrerhandreichungen erarbeiteten interpretatorischen Ansätze und die unterrichtsdidaktischen Empfehlungen. Sie enthalten vor allem im Zusammenhang mit der Novelle Der Schimmelreiter hochinteressante und noch auszuwertende Aspekte; immerhin blickt man bei diesem Text mittlerweile auf 70 bis 80 Jahre der Vermittlung in deutschen Schulen zurück.

Die Forschungsliteratur ist in der Bibliographie im Materialienteil zusammengefaßt, geordnet nach der Kapitelgliederung des $\mathrm{Bu}$ ches und innerhalb dieser Gliederung chronologisch nach dem Erscheinungsjahr; nur die Briefwechsel werden alphabetisch nach den Adressaten verzeichnet. Im Text zitierte Literatur, die über den engeren thematischen Rahmen hinausreicht, steht in der Bibliographie am Ende der jeweiligen Kapitel.

Danken möchte ich an dieser Stelle all jenen, die meine Arbeit an diesem Band begleitet haben, im besonderen meinen Freunden und Kollegen Christine und Volker Giel, Michael Masanetz und Elke Richter. 